\title{
Lipid-Lowering Drug Therapy: Critical Approach for Implementation in Clinical Practice
}

\author{
Maddalena Rossi $^{1}$ (1) $\cdot$ Enrico Fabris $^{1} \cdot$ Davide Barbisan $^{1} \cdot$ Laura Massa $^{1} \cdot$ Gianfranco Sinagra $^{1}$
}

Accepted: 11 August 2021 / Published online: 13 September 2021

(c) The Author(s) 2021

\begin{abstract}
Increased levels of low-density lipoprotein cholesterol (LDL-C) are recognized as a primary risk factor for atherosclerotic cardiovascular disease, which remains the leading cause of death worldwide. Lowering LDL-C levels clearly reduces the risk of cardiovascular events, with benefits related to both absolute reduction and duration of treatment; however, a threshold below which low LDL-C levels can be dangerous has never been established. Since the discovery of statins, cardiovascular research has focused on developing new lipid-lowering agents. Ezetimibe and proprotein convertase subtilisin-kexin type 9 inhibitors have been found to further reduce LDL-C values and subsequent cardiovascular risk. Novel recently approved inclisiran and bempedoic acid, currently being tested in cardiovascular outcomes studies, are further expanding our pharmacological armamentarium, enabling the clinician to diminish residual risk related to LDL-C. Moreover, new agents are paving the way to successful treatment of homozygous familial hypercholesterolemia. This review summarizes the main characteristics of current and emerging lipid-lowering therapies to assist with comprehensive evidence-based decision making.
\end{abstract}

\section{Introduction: Why Do We Need New Lipid-Lowering Therapies?}

Accounting for $30 \%$ of the world's total deaths, atherosclerotic cardiovascular disease (ASCVD) is considered the leading cause of morbidity and mortality all over the world [1]. In Europe, it causes more than 4 million deaths annually, with an estimated global economic cost of $€ 196$ billion per year, in terms of direct healthcare costs, loss of work productivity, and informal family care [2].

It is well-known that low-density lipoprotein cholesterol (LDL-C) represents the key initiating event in atherogenesis as it enters the subendothelial space, accumulates in the

Gianfranco Sinagra

gianfranco.sinagra@asugi.sanita.fvg.it

Maddalena Rossi

maddalenarossi93@gmail.com

Enrico Fabris

enrico.fabris@hotmail.it

Davide Barbisan

barbisand@gmail.com

Laura Massa

laura.massa@asugi.sanita.fvg.it

1 Cardiovascular Department, Azienda Sanitaria Universitaria Giuliano Isontina and University of Trieste, Trieste, Italy arterial wall, and promotes a local inflammatory response [3]. A consistent body of evidence from randomized controlled trials (RCTs) confirms that elevated LDL-C is associated with the highest odds ratio for ASCVD among all modifiable cardiovascular risk factors [4], with an "effect size" proportional to both LDL-C blood levels and the total time of exposure. Moreover the positive association between ASCVD and LDL-C extends to even very low levels of LDL-C [5], and lowering LDL-C and apolipoprotein $\mathrm{B}(\mathrm{apoB})$ reduces the risk of major atherosclerotic vascular events, with benefits directly and positively correlated with the incrementally achieved absolute LDL-C reduction and duration of treatment [6-8]. Most importantly, a threshold below which LDL-C reduction is dangerous has never been detected, so there is no lower limit for achievable LDL-C values [7].

Given this, the 2019 European Society of Cardiology (ESC)/European Atherosclerosis Society (EAS) guidelines for the management of dyslipidemias have further lowered LDL-C goals for each cardiovascular risk class, thus paving the way for implementation of new pharmacological approaches. 


\section{Current Approach}

Current European guidelines for the management of dyslipidemias recommend high-intensity statins prescribed up to the highest tolerated dose as first-line therapy to reach the LDL-C value set for the specific level of risk. If this goal is not achieved, combination with ezetimibe is recommended [7]. If statin plus ezetimibe is still insufficient to reach recommended LDL-C levels, adding a proprotein convertase subtilisin/kexin type 9 inhibitor (PCSK9i) may be considered in very high-risk patients for primary prevention and is strongly recommended for secondary prevention and in high-risk patients with familial hypercholesterolemia (FH) [7]. If a statin-based regimen is not tolerated at any dosage, ezetimibe and eventually PCSK9i should be considered [7].

\subsection{Statins}

Statins are the universally recognized cornerstone of the lipid-lowering pharmacological armamentarium, since they have been shown to reduce rates of cardiovascular events [9]. They reduce the synthesis of cholesterol in the liver by competitively inhibiting the enzyme HMG-CoA reductase, thus promoting increased expression of LDL receptors (LDLRs) on the surface of hepatocytes and consequent increased uptake of LDL-C from the blood [10]. The degree of LDL-C reduction is dose dependent and patient dependent: high-intensity regimens (e.g., atorvastatin $40-80 \mathrm{mg} /$ day) have been shown to reduce LDL-C by $\geq 50 \%$ from baseline, whereas moderate-intensity therapy (e.g., atorvastatin 10-20 mg) is expected to reduce LDL-C by $30-50 \%$, with remarkable interindividual variation explained by differences in both compliance and genetic backgrounds [7]. In the CCT (Cholesterol Treatment Trialists) meta-analysis, for each $38.7 \mathrm{mg} / \mathrm{dL}$ reduction in LDL$\mathrm{C}$, statins reduced major cardiovascular events by $22 \%$ and total mortality by $10 \%$ over 5 years [9], with an absolute risk reduction proportional to absolute baseline risk.

In the real-world clinical setting, the use of statins at their highest doses is limited by a non-negligible frequency of side effects, mostly represented by musclerelated symptoms without significant increases in creatine kinase (CK), with an incidence as high as $29 \%$, which is greater than that reported in RCTs [11]. Moreover, $0.1 \%$ of patients may experience serious muscle injury, and $0.2 \%$ of patients experience an important increase in transaminases and new-onset diabetes.

Observational data suggest that approximately half of patients in whom statin therapy is initiated do not have optimal adherence to treatment or will discontinue it within 1 year [12], thus substantially affecting the achievement of LDL-C target levels. Moreover, in patients with persistent high LDL-C blood levels despite maximum tolerated statin doses, a substantially elevated risk for cardiovascular events remains [13].

\subsection{Ezetimibe}

Ezetimibe inhibits the intestinal sterol transporter NiemannPick C1-like 1 (NPC1L1), thus reducing intestinal absorption of dietary and biliary cholesterol and the consequent amount of cholesterol delivered to the liver, in response to which the liver upregulates LDLR expression, leading to increased clearance of LDL-C from the blood [14]. Ezetimibe is generally well-tolerated and does not require dose adjustment in patients with mild to severe chronic kidney disease (CKD). Moreover, when added to statin therapy, ezetimibe does not appear to increase the incidence of elevated CK levels [7].

Ezetimibe at a daily oral dose of $10 \mathrm{mg}$ reduces LDL-C by $15-22 \%$ as monotherapy and by an additional $21-27 \%$ when added to ongoing statin therapy [15], with relatively high interindividual variations. Three large trials have addressed ezetimibe cardiovascular outcomes. The SEAS (Simvastatin and Ezetimibe in Aortic Stenosis) and SHARP (Study of Heart and Renal Protection) trials compared simultaneous ezetimibe/simvastatin treatment versus placebo in 1873 patients with aortic stenosis and 9270 patients with CKD, respectively $[16,17]$. The combination of ezetimibe/ simvastatin significantly reduced the incidence of ischemic cardiovascular events by $22 \%$ and $17 \%$, respectively [16, 17]. The most recent IMPROVE-IT (Improved Reduction of Outcomes: Vytorin Efficacy International Trial) randomized 18,144 patients with a history of acute coronary syndrome (ACS) to receive statin $40 \mathrm{mg} /$ placebo or statin $40 \mathrm{mg} /$ ezetimibe $10 \mathrm{mg}$ [18]. The average LDL-C was $70 \mathrm{mg} / \mathrm{dL}$ in the statin/placebo group versus $54 \mathrm{mg} / \mathrm{dL}$ in the statin/ ezetimibe arm. At the median follow-up of 6 years, the incremental lowering of LDL-C with statin/ezetimibe translated to a reduction of major atherosclerotic events (absolute risk difference $2.0 \%$, hazard ratio [HR] $0.94 ; 95 \%$ confidence interval $[\mathrm{CI}] 0.89-0.99 ; P=0.016)$ in the absence of remarkable adverse events compared with the control arm [18].

Even though statistical models show that ideal treatment with high-intensity statin plus ezetimibe should leave only $14 \%$ of patients with ASCVD far from LDL-C therapeutic targets defined by ESC/EAS guidelines [19], only approximately $30 \%$ of these patients achieve acceptable LDL-C levels in everyday clinical practice because of different genetic backgrounds, suboptimal compliance with a daily regimen, and low tolerance of side effects [20]. 


\subsection{Proprotein Convertase Subtilisin-Kexin Type 9 Inhibitors}

In recent years, PCSK9i have emerged as a new and effective lipid-lowering therapy (LLT). PCSK9 is a circulating liver-synthesized serine-protease that binds LDLR on the hepatocyte surface, promoting their lysosomal degradation, and acting as one of the most important regulators of LDL-C metabolism [21, 22]. The two available drugs of this class, evolocumab and alirocumab, are fully human monoclonal antibodies (mAbs) sequestering PCSK9 in the reticuloendothelial system, preventing the binding of PCSK9 with LDLR, and therefore upregulating LDLR expression and increasing LDL-C clearance [21, 22]. Of note, a third partially humanized PCSK9i mAb, bococizumab, was studied in the SPIRE program but prematurely discontinued after the detection of neutralizing antibodies against the drug, causing a progressive reduction of LDL-C-lowering effectiveness [23].

Both alirocumab and evolocumab need to be administered by subcutaneous injection once or twice monthly. Clinical trials showed their efficacy in significantly lowering LDL-C levels by approximately $60 \%$, either alone or in addition to a background therapy with statins and/or ezetimibe, without safety concerns [24]. Indeed, when added to maximally tolerated statins, they reduced LDL-C by $30 \%$ more than ezetimibe. Moreover, unlike statins, they also reduced lipoprotein(a) plasma levels by $35 \%$ [7].

Two large RCTs recently showed that the addition of PCSK9i mAbs to statin therapy provided a further reduction in ASCVD risk: the FOURIER (Further Cardiovascular Outcomes Research with PCSK9 Inhibition in Subjects with Elevated Risk) [25] trial enrolled patients with coronary heart disease (CHD)/peripheral artery disease (PAD)/previous stroke, and the ODYSSEY (Evaluation of Cardiovascular Outcomes After an Acute Coronary Syndrome During Treatment with Alirocumab) [26] trial enrolled patients with recent ACS and LDL-C levels $\geq 70 \mathrm{mg} / \mathrm{dL}$ despite maximally tolerated statin doses. After a median follow-up of 2.2 and 2.8 years, respectively, treatment with evolocumab or alirocumab was associated with a significant reduction of the risk of ischemic cardiovascular events (HR $0.85 \%$ in both trials) [25, 26]. Moreover, according to some authors, since the evidence from statin trials highlights that the clinical benefits of LLTs is greater after the first year of treatment, these two trials, with their relatively short follow-up, could have underestimated the beneficial effects of long-term therapy [27]. On the other hand, given the high lipid-lowering efficacy of PCSK9i mAbs, how much LDL-C should or can be safely lowered remains under debate. In patients from the FOURIER trial who had a baseline LDL-C $<70 \mathrm{mg} /$ $\mathrm{dL}$, evolocumab lowered LDL-C to $21 \mathrm{mg} / \mathrm{dL}$, reducing the risk of death, myocardial infarction (MI), or stroke by
$30 \%$ compared with placebo (HR $0.70 ; 95 \%$ CI $0.48-1.01$ ) [28]. In addition, a prespecified secondary analysis from the FOURIER trial described a monotonic relationship between lowered LDL-C and a lower risk of major cardiovascular outcomes, extending down to LDL-C levels of $<10 \mathrm{mg} / \mathrm{dL}$ without significant associations with multiple safety outcomes [29].

Importantly, PCSK9i mAbs have an excellent safety profile, since the most frequently reported adverse events are only mild-to-moderate injection-site reactions and flu-like symptoms [25, 26], whereas the initially suspected neurocognitive function changes were not confirmed in the EBBINGHAUS (Evaluating PCSK9 Binding Antibody Influence on Cognitive Health in High Cardiovascular Risk Subjects) study [30].

As these RCTs did not include patients with severe renal impairment, evolocumab and alirocumab should not currently be used in patients with estimated glomerular filtration rate $(\mathrm{eGFR})<20$ and $30 \mathrm{~mL} / \mathrm{min} / 1.73 \mathrm{~m}^{2}$, respectively $[25,26]$.

Although PCSK9i mAbs provide large LDL-C reductions, which is a valuable means of reducing the ASCVD burden, they are still not widely used in everyday clinical practice. This could be related to the significant bureaucratic requirements driven by healthcare budget impact concerns. Indeed, considering an average yearly cost of \$5400-5800 (US \$, year 2020 values) per patient, they are considered cost effective only in patients at very high risk of ASCVD [31, 32]. Finally, despite their good tolerability, registry-derived adherence data are unsatisfactory, reporting a discontinuation rate of $35-40 \%$ after 6 months, probably because of the need for subcutaneous administration, which might reduce patient adherence [31].

\section{New Emerging Therapies}

\subsection{Bempedoic Acid}

Bempedoic acid (8 hydroxy-2,2,14,14 tetra-methylpentadecanedioic acid) is a recently approved orally available prodrug that is rapidly adsorbed in the small intestine [33]. In the liver, it is converted to its active coenzyme A conjugate by a hepatic-specific isoform of the very-long chain acyl-CoA synthetase-1, thus ensuring no interference with cholesterol biosynthesis in other tissues (e.g., muscles) and potentially avoiding some of the systemic effects associated with statins. The bempedoic acid-CoA competitively inhibits ATP-citrate lyase, a cytosolic enzyme acting in the cholesterol biosynthesis pathway upstream of the 3-hydroxy 3-methylglutaryl-coenzyme A (HMG-CoA) reductase, the target of statins [33, 34]. As such, it decreases intrahepatic 
cholesterol synthesis, upregulating LDLR expression and subsequent circulating LDL-C clearance [34].

In CLEAR (Cholesterol Lowering via Bempedoic Acid, an ACL-Inhibiting Regimen) Tranquility, the first phase III RCT of bempedoic acid, a dose of $180 \mathrm{mg}$ daily added to ezetimibe lowered LDL-C by $28.5 \%$ in patients with hypercholesterolemia at high cardiovascular risk and unable to tolerate optimal statin therapy [35]. Of note, the efficacy of bempedoic acid was greater in patients not treated with statins, probably because the two drugs target the same pathway [33]. In the later CLEAR Serenity trial, bempedoic acid reduced LDL-C by $21.4 \%(p<0.001)$ in patients with statin intolerance regardless of baseline LDL-C or concomitant LLT (also including PCSK9i mAbs) [36].

CLEAR Harmony, the largest trial to date, assessed the safety and efficacy of this drug in 2230 patients with ASCVD and/or heterozygous FH (HeFH) and LDL-C of $\geq 70 \mathrm{mg} / \mathrm{dL}$ while receiving maximally tolerated statin [37]. At week 12, bempedoic acid reduced the mean LDL-C blood level by $18 \%$ versus placebo $(95 \%$ CI -20.0 to -16.1 ; $p<0.001$ ), regardless of the intensity of background statin therapy, with the beneficial effect substantially stable until week 52 and without a higher incidence of overall adverse events than placebo [37]. However, in CLEAR Harmony, the primary endpoint was a safety endpoint (incidence of overall adverse events and changes in safety laboratory variables), and the percentage change in LDL-C level was only the main secondary endpoint [37]. CLEAR Wisdom, a smaller RCT with similar design but primary efficacy endpoint of percentage change in LDL-C level at week 12, reported similar results [38].

The CLEAR RCTs also showed that bempedoic acid reduced triglycerides, total cholesterol, apoB, and C-reactive protein (CRP), suggesting a possible anti-inflammatory effect that could contribute to cardiovascular risk reduction [39].

It is noteworthy that RCTs and their subsequent metaanalyses reported rates of muscle-related adverse events in patients treated with bempedoic acid similar to those in the placebo group, thanks to the selective activation of the prodrug in the liver [33]. This evidence makes this drug the perfect alternative in patients who do not tolerate statins because of clinically relevant muscular side effects. The only important adverse event emerging during the treatment with bempedoic acid was a significant increase in plasma uric acid and risk of acute gout, mostly occurring in patients with a previous history of hyperuricemia [37]. This has been attributed to the competition between a metabolite of bempedoic acid and uric acid for the same renal transporter [37]. Moreover, bempedoic acid weakly inhibits organic anion-transporting peptide 1B1 and 1B3, thus increasing plasma concentrations of drugs such as bosentan, fimasartan, asunaprevir, glecaprevir, grazoprevir, and some statins. For example, because of the described twofold increase in simvastatin exposure caused by bempedoic acid, it cannot be used with a simvastatin dose $>20 \mathrm{mg} /$ day $[40,41]$.

Unlike statins, ezetimibe, and PCSK9i mAbs, no evidence on the efficacy of bempedoic acid treatment in reducing cardiovascular morbidity and mortality are yet available (Table 1). On this basis, the ongoing CLEAR Outcomes RCT, including 13,000 patients followed for about 4 years, is testing the effect of bempedoic acid on cardiovascular outcomes in patients with a history of statin intolerance [42].

In February 2020 and March 2020, respectively, the US FDA and the European Medicines Agency (EMA) approved the use of bempedoic acid in adults with primary hypercholesterolaemia (heterozygous familial and nonfamilial) or mixed dyslipidemia, as an adjunct to diet. Bempedoic acid can be used

(1) in combination with a statin and other LLTs in patients unable to reach LDL-C goals with the maximum tolerated statin dose and

(2) alone or in combination with ezetimibe in patients who are statin intolerant or for whom a statin is contraindicated $[40,41]$.

As the RCTs did not include patients with severe renal impairment, bempedoic acid should not currently be used in patients with $\mathrm{eGFR}<30 \mathrm{~mL} / \mathrm{min} / 1.73 \mathrm{~m}^{2}$ [33].

\subsection{Inclisiran}

The last frontier in LLT strategies is represented by inclisiran, which uses an alternative approach to regulating the PCSK9 pathway. It is a double-stranded small-interfering RNA (siRNA) that inhibits PCSK9 synthesis by binding and then degrading the specific messenger RNA (mRNA), thus preventing its translation into the PCSK9 protein [43]. Similarly to PCSK9i mAbs, this results in downregulation of LDLR lysosomal catabolism, supporting more efficient clearance of LDL-C from the bloodstream. Thanks to its conjugation to the triantennary $\mathrm{N}$-acetylgalactosamine (GalNac), inclisiran is specifically recognized by a receptor highly expressed on hepatocytes, thus ensuring liver selectivity [43]. In the liver, it is trapped in the endosomes, creating intracellular depots from which it can be slowly released over time, allowing a durable effect. Moreover, its chemical structure means it is stable at room temperature, unlike PCSK9i mAbs, which require refrigeration [42].

Inclisiran was evaluated in the ORION programs, including 11 RCTs summarized in Table 2. ORION-9, ORION-10, and ORION-11 were phase III randomized, placebo-controlled trials that studied the efficacy and 


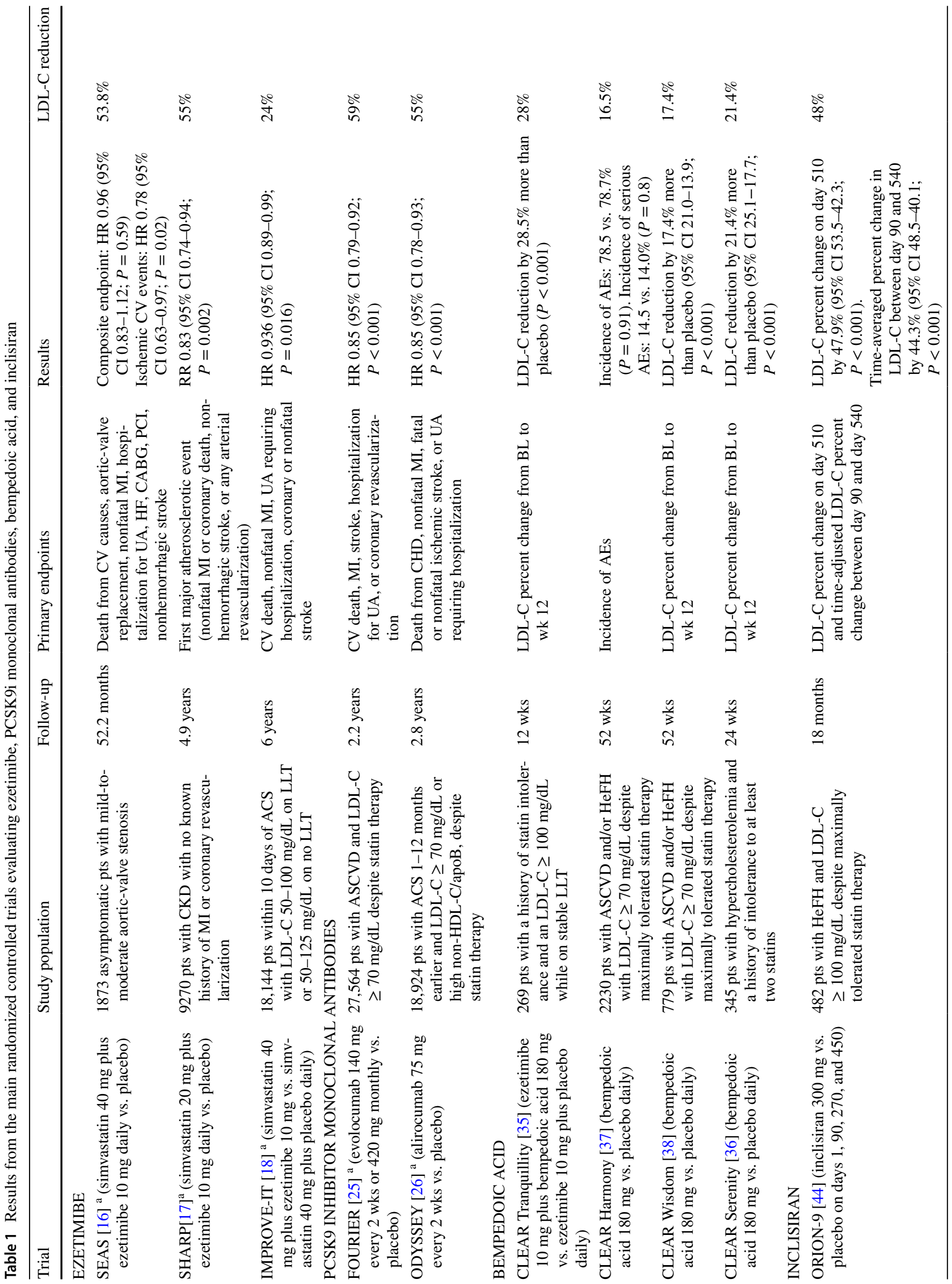




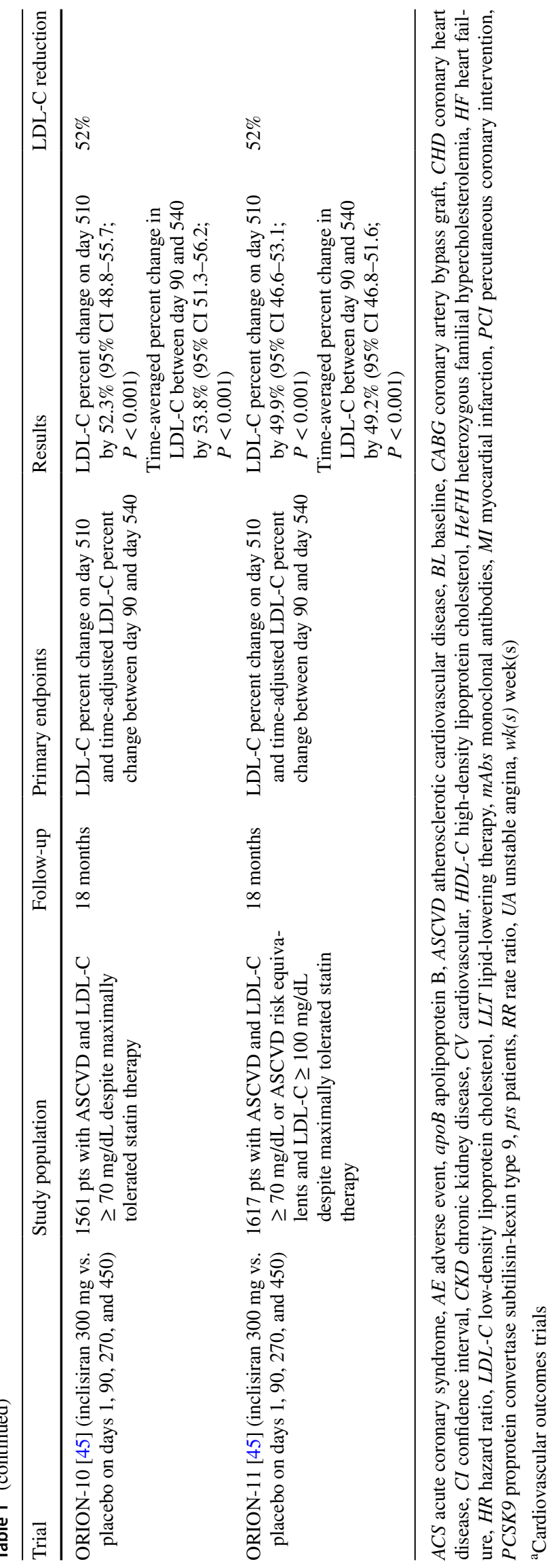


Table 2 Randomized controlled trials of ORION development program for inclisiran

\begin{tabular}{|c|c|c|c|}
\hline Trial & Description & Patients $(n)$ & Follow-up \\
\hline ORION-1 & Phase II dose finding trial & 501 & 360 days \\
\hline ORION-2 & Pilot study enrolling patients with $\mathrm{HoFH}$ & 4 & 300 days \\
\hline ORION-3 & Open-label extension study of ORION-1, compared with evolocumab & 374 & 4 years \\
\hline ORION-4 & Cardiovascular outcomes phase III trial & 15,000 & $\begin{array}{l}\text { Event-driven } \\
\quad \text { (median dura- } \\
\text { tion } \geq 5 \text { years) }\end{array}$ \\
\hline ORION-5 & Phase III trial enrolling patients with $\mathrm{HoFH}$ & 45 & 2 years \\
\hline ORION-6 & $\begin{array}{l}\text { Supporting single-dose, open-label, parallel-group study, designed to assess inclisiran pharma- } \\
\text { cology in subjects with mild or moderate hepatic impairment compared with subjects with } \\
\text { normal liver function }\end{array}$ & 24 & 180 days \\
\hline ORION-7 & $\begin{array}{l}\text { Supporting single-dose, open-label, parallel-group study designed to evaluate inclisiran pharma- } \\
\text { cology in subjects with renal impairment }\end{array}$ & 31 & 180 days \\
\hline ORION-8 & Phase III extension study of ORION-9, 10, and 11 & 3600 & 3 years \\
\hline ORION-9 & Phase III trial designed to assess efficacy and safety of inclisiran in patients with $\mathrm{HeFH}$ & 482 & 540 days \\
\hline ORION-10 & Phase III trial designed to assess efficacy and safety of inclisiran in patients with ASCVD (USA) & 1561 & 540 days \\
\hline ORION-11 & $\begin{array}{l}\text { Phase III trial designed to assess efficacy and safety of inclisiran in patients with ASCVD or } \\
\text { ASCVD equivalents (Europe-South Africa) }\end{array}$ & 1617 & 540 days \\
\hline
\end{tabular}

$A S C V D$ atherosclerotic cardiovascular disease, $\mathrm{HeFH}$ heterozygous familial hypercholesterolemia, HoFH homozygous familial hypercholesterolemia

safety of inclisiran $300 \mathrm{mg}$, administered by subcutaneous injections at baseline, after 3 months, and then every 6 months, in patients with HeFH, ASCVD, and ASCVD or ASCVD risk equivalents, respectively, with a follow-up of 540 days [44].

ORION-9 enrolled 482 patients with HeFH and LDL-C blood levels $\geq 100 \mathrm{mg} / \mathrm{dL}$ despite maximally tolerated statin with or without ezetimibe [44]. The mean baseline level of LDL-C was $153 \mathrm{mg} / \mathrm{dL}$, although $90 \%$ of patients were on treatment with statins (75\% with high-intensity statins). At 17 months, inclisiran was effective in lowering LDL-C by $47.9 \%$ compared with placebo $(95 \%$ CI -53.5 to -42.3 ; $p<0.001)$ with a time-averaged percent change between day 90 and day 540 of $44.3 \%$ [44]. Interestingly, an exploratory analysis showed robust reductions in LDL-C levels in all genotypes of $\mathrm{FH}$, with an extraordinary value in patients with PCSK9 gain-of-function variant [44].

ORION-10 and ORION-11 were conducted with identical protocols, in the USA and Europe/South Africa, respectively. ORION-10 enrolled 1561 patients with ASCVD and LDL-C $\geq 70 \mathrm{mg} / \mathrm{dL}$, whereas ORION-11 enrolled 1617 patients with ASCVD or ASCVD risk equivalent (type 2 diabetes mellitus, $\mathrm{FH}$, a 10-year risk of cardiovascular event $\geq 20 \%$ as assessed by the Framingham Risk Score for Cardiovascular Disease), and LDL-C $\geq 70$ or $\geq 100 \mathrm{mg} / \mathrm{dL}$, respectively [45].

All the patients were on stable LLT with statins at the maximum tolerated dose (more than $70 \%$ on high-intensity statin) with or without ezetimibe. At day 510, inclisiran reduced LDL-C levels by $52.3 \%$ (95\% CI -48.8 to -55.7 ; $p<0.001)$ in the ORION-10 trial and by $49.9 \%$ (95\% CI -46.6 to $-53.1 ; p<0.001)$ in the ORION-11 trial, with corresponding time-adjusted reductions of 53.8 and $49.2 \%$ [45].

Of note, patients receiving treatment with PCSK9i mAbs within 90 days before screening were excluded, as were those with eGFR $<30 \mathrm{~mL} / \mathrm{min} / 1.73 \mathrm{~m}^{2}$ [44, 45].

Regarding the secondary endpoints, all three trials showed a statistically significant reduction in total cholesterol, triglycerides, lipoprotein(a), PCSK9, and apoB levels, the clinical meaning of which is not yet totally clear $[44,45]$.

In the safety analysis, all three trials reported similar adverse event and serious adverse event rates in the experimental and control groups, except for mild-to-moderate injection-site reactions, which were more frequent with inclisiran than with placebo [44, 45]. None of these trials reported significant changes in liver/kidney function tests, CRP, or platelet count, as described during other therapies with siRNAs. Antidrug antibodies were detected in $2.5 \%$ of treated patients but were at low titers, often transient, and not associated with pharmacologic changes [45]. However, additional data on long-term safety and tolerability of inclisiran will be provided by results of ORION-8, the 3-year extension study of ORION-9, ORION-10, and ORION-11.

Interestingly, both ORION-10 and ORION-11 included a prespecified cardiovascular exploratory endpoint of cardiac death, nonfatal MI, and stroke, which was reported with lower frequency in the inclisiran group (7.4-7.8\% vs. $10.210 .3 \%$ in the placebo group) [45]. These numbers are too small to draw meaningful conclusions about any 
potential benefits of inclisiran on cardiovascular morbidity and mortality, a question that is being tested in ORION-4, the ongoing cardiovascular outcomes trial including 15,000 patients [45].

In summary, inclisiran, given every 6 months on top of maximum tolerated guideline-recommended statin treatment, was effective in lowering LDL-C by $50 \%$ and had a good safety profile and negligible interindividual variability. This reduction remained stable within the dosing interval. Inclisiran appeared to have the same efficacy as PCSK9i mAbs but a lower "injection burden," promising greater medication adherence. No information is yet available about the price of inclisiran; however, an initial analysis by the Institute for Clinical and Economic Review indicated that it should cost between \$US3600 and 6000 per year (year 2020 values) to meet cost-effectiveness standards [46].

On December 2020, the EMA approved the use of inclisiran for the treatment of adults with primary hypercholesterolemia (heterozygous familial and nonfamilial) or mixed dyslipidemia as an adjunct to diet [47]. Inclisiran can be used

(1) in combination with a statin (with or without other LLT) in patients unable to reach LDL-C goals with the maximally tolerated dose of a statin and

(2) alone or in combination with other LLT in patients who are statin intolerant or for whom a statin is contraindicated [47].

Table 1 presents the results from the main RCTs evaluating ezetimibe, PCSK9i mAbs, bempedoic acid, and inclisiran.

\subsection{Lomitapide and Mipomersen in Homozygous Familial Hypercholesterolemia}

Homozygous $\mathrm{FH}(\mathrm{HoFH})$, one of the most common inherited metabolic disorders, is a life-threatening condition characterized by markedly elevated circulating LDL-C, leading to premature and progressive ASCVD before the age of 20 years [48]. It is most commonly caused by loss-of-function mutations in LDLR genes, meaning that conventional LLTs, which generally upregulate hepatic LDLRs, are often inadequate, and LDL apheresis remains the standard of care. Mipomersen and lomitapide, which target other cellular pathways, are promising new agents for this specific setting.

Mipomersen is an antisense oligonucleotide (ASO) administered via subcutaneous injection that reduces hepatic very-low-density lipoprotein (VLDL) assembly by preventing apoB 100 protein translation [7]. A double-blind, placebo-controlled registration study showed that mipomersen reduced LDL-C by approximately $25 \%$, but it has been associated with significant hepatotoxicity and steatosis. The FDA approved mipomersen for the treatment of $\mathrm{HoFH}$, but the drug is not approved in Europe [49].

Lomitapide is an orally administered drug that inhibits the microsomal triglyceride transfer protein and prevents the assembly of VLDL in the liver and chylomicrons in the intestine, thereby lowering circulating LDL-C. An open-label, single-arm phase III study observed that lomitapide $40 \mathrm{mg} /$ day reduced LDL-C by $50 \%$ (95\% CI $62-39 ; p<0.0001)$ in 29 patients with $\mathrm{HoFH}$, decreasing the frequency of apheresis [50]. Interestingly, the significant LDL-C reduction remained stable in the 5-year extension study. The most reported adverse events were gastrointestinal disorders, liver transaminase elevation, and transitory hepatic fat accumulation, managed by dose reduction or temporary interruption but not requiring permanent discontinuation of the drug [50]. Lomitapide, the effect of which on cardiovascular morbidity and mortality has not yet been determined, is actually indicated as an adjunct to a low-fat diet and other LLT with or without LDL apheresis in patients with HoFH [51].

\section{Other Approaches to Low-Density Lipoprotein Cholesterol Lowering}

Other interesting emerging therapies target angiopoietinlike 3 (ANGPTL3), a potent inhibitor of lipoprotein lipase, whose loss of function is associated with decreased plasma levels of triglycerides, LDL-C, and elevated high-density lipoprotein cholesterol (HDL-C) [49]. Evinacumab is a fully human $\mathrm{mAb}$ that binds circulating ANGPTL3 and was reported to be effective in reducing triglycerides and LDL-C in healthy human volunteers and in patients with $\mathrm{HoFH}$. A GalNAc-modified ASO targeting ANGPTL3, named IONIS-ANGPTL3-LRx, is undergoing phase II trials and was shown to reduce atherogenic lipoproteins in human volunteers with elevated triglycerides [52].

More controversial is the history of the inhibitors of the enzyme cholesteryl ester transfer protein (CETP), which normally transfers cholesterol from HDL-C to VLDL or LDL. Many CETP inhibitors, although providing higher HDL-C levels and lower circulating LDL-C, have failed in clinical trials, either causing an increase in deaths (torcetrapib [53]) or resulting in no meaningful clinical improvement (dalcetrapib, evacetrapib) [54]. Finally, the REVEAL (Randomized Evaluation of the Effects of Anacetrapib through Lipid modification) study, involving 30,449 patients with ASCVD randomized to receive anacetrapib $100 \mathrm{mg}$ daily or placebo on top of statin therapy, demonstrated a highly significant reduction (rate ratio $0.91 ; p<0.004$ ) in the composite primary endpoint of coronary death, MI, or coronary revascularization [55]. The average increase in HDL-C was $104 \%$, with a decrease in LDL-C by $17 \%$ [55]. Despite these successful results, the development of anacetrapib was 
halted because analysts considered the benefit, albeit statistically significant, insufficient to justify the pursuit of regulatory approval [56]. Moreover, some safety issues were raised by some evidence that anacetrapib accumulates in fat tissue with prolonged dosing [56].

More promising developments may be expected for the CETP inhibitor obicetrapib, an orally administered smallmolecule drug previously known as TA-8995 and later as AMG 899. In the phase IIb TULIP trial, which enrolled 364 subjects with hypercholesterolemia (mean baseline LDL-C $140 \mathrm{mg} / \mathrm{dL})$, optimal TA-8895 monotherapy $(10 \mathrm{mg})$ produced LDL-C and apoB reductions of 45 and $35 \%$, respectively, and an HDL-C increase of $180 \%$ over a 12 -week dosing period [57]. In combination with statins, TA-8995 was associated with an additional decrease in LDL-C of $40-50 \%$ and in apoB of $30-35 \%$. In terms of safety considerations, TA-8995 was well-tolerated and did not accumulate during the dosing period or 8 weeks after the cessation of the study period [57]. In 2017, the obicetrapib development program was discontinued for strategic reasons; its licenses were later purchased by another pharmaceutical company, and two phase II placebo-controlled, double-blind, randomized studies are currently investigating the use of the drug.

The first is a phase II dose-finding study to evaluate the efficacy, safety, and tolerability of obicetrapib as an adjunct to high-intensity statin therapy for 8 weeks in patients with LDL-C $>70 \mathrm{mg} / \mathrm{dL}$ (ROSE trial; NCT04753606) [58]. The second, registered as OCEAN (NCT04770389 and 2019004935-22), has enrolled patients with mild dyslipidemia (LDL-C $>100$ and $<175 \mathrm{mg} / \mathrm{dL}$ ) with the aim of evaluating the efficacy, safety, and tolerability of obicetrapib and ezetimibe combination therapy [59]. In both trials, the primary endpoint is percent change in LDL-C after 8 weeks of treatment, and secondary endpoints are percent change in apoB, HDL-C, and non-HDL-C [58, 59].

In addition, obicetrapib will be tested in a comprehensive phase III clinical trial planned to start at the end of 2021. If the trial confirms its good efficacy, even in monotherapy, and tolerability, obicetrapib will become another effective strategy for patients whose lipid levels are not well-controlled on statins and patients who are statin intolerant.

\section{Drugs Under Development}

Other promising approaches to PSCK9 inhibition are now under investigation, including lerodalcibep, gene editing using CRISPR-Cas9 techniques, and vaccination-like approaches, with studies still in animal phases [60, 61].

Lerodalcibep (formerly LIB003) is a recombinant fusion protein of a PCSK9-binding domain (adnectin) and human serum albumin, which increases its half-life to 12-15 days and allows monthly administration as a small-volume $(1 \mathrm{~mL})$ injection [62]. In a phase II study enrolling patients with LDL-C $>80 \mathrm{mg} / \mathrm{dL}$ despite maximally tolerated statin, lerodalcibep $300 \mathrm{mg}$ administered monthly was effective in lowering LDL-C by more than $70 \%$ at the end of the 12 -week study, with an incidence of adverse events similar to that with placebo [63]. A subsequent extension study confirmed these data, showing a stable mean $60 \%$ reduction in LDL-C through 36 weeks [63].

On this basis, a clinical development program focusing on HoFH, ASCVD, and high-risk primary prevention is ongoing. It consists of six phase III trials, summarized in Table 3.

Another attractive strategy is represented by vaccines against PCSK9 that should trigger the generation of host anti-PCSK9 antibodies, neutralizing PCSK9/LDLR interactions. A novel antiPCSK9 vaccine formulation, called liposomal immunogenic fused PCSK9-tetanus peptide plus alum adjuvant (L-IFPTA), was recently designed [64]. The efficacy of L-IFPTA vaccine, containing two different epitopes belonging to PCSK9 and tetanus toxin proteins, has been shown in different animal models, inducing the production of long-lasting functional anti-PCSK9 antibodies in BALB/c mice and reducing LDL-C and VLDL-C by up to 51.7 and $19.2 \%$ in C57BL/6 mice, without significant adverse events [65].

Although this field of research remains in its very early states, it is promising, especially for the future possibility of avoiding frequent administrations, such as required by PCSK9i mAbs. Given this encouraging preclinical evidence, the L-IFPTA formulation is expected to enter phase I testing by the end of 2021 .

\section{Targeting the Optimal Patients: Who Could Benefit The Most?}

The recently approved bempedoic acid and inclisiran will soon become part of the everyday pharmacologic armamentarium, increasing clinicians' abilities to implement LLT (Fig. 1; Table 4). Given that statins and ezetimibe represent the universally recommended first step in every LLT strategy, how to select these new drugs when further LDL-C reduction is needed has not yet been established.

The clinician should take into account the patient's age and life expectancy, baseline LDL-C level, need for primary or secondary prevention, expected therapeutic compliance, and the patient's personal preference for oral or subcutaneous administration.

Given their different efficacies, bempedoic acid could be used when a $20 \%$ further reduction in LDL-C levels is needed, and PCSK9i (mAbs or inclisiran), with their higher efficacy but higher prices, could be used in very high LDL-C blood levels despite treatment, especially in patients with recent cardiovascular events or at very high 


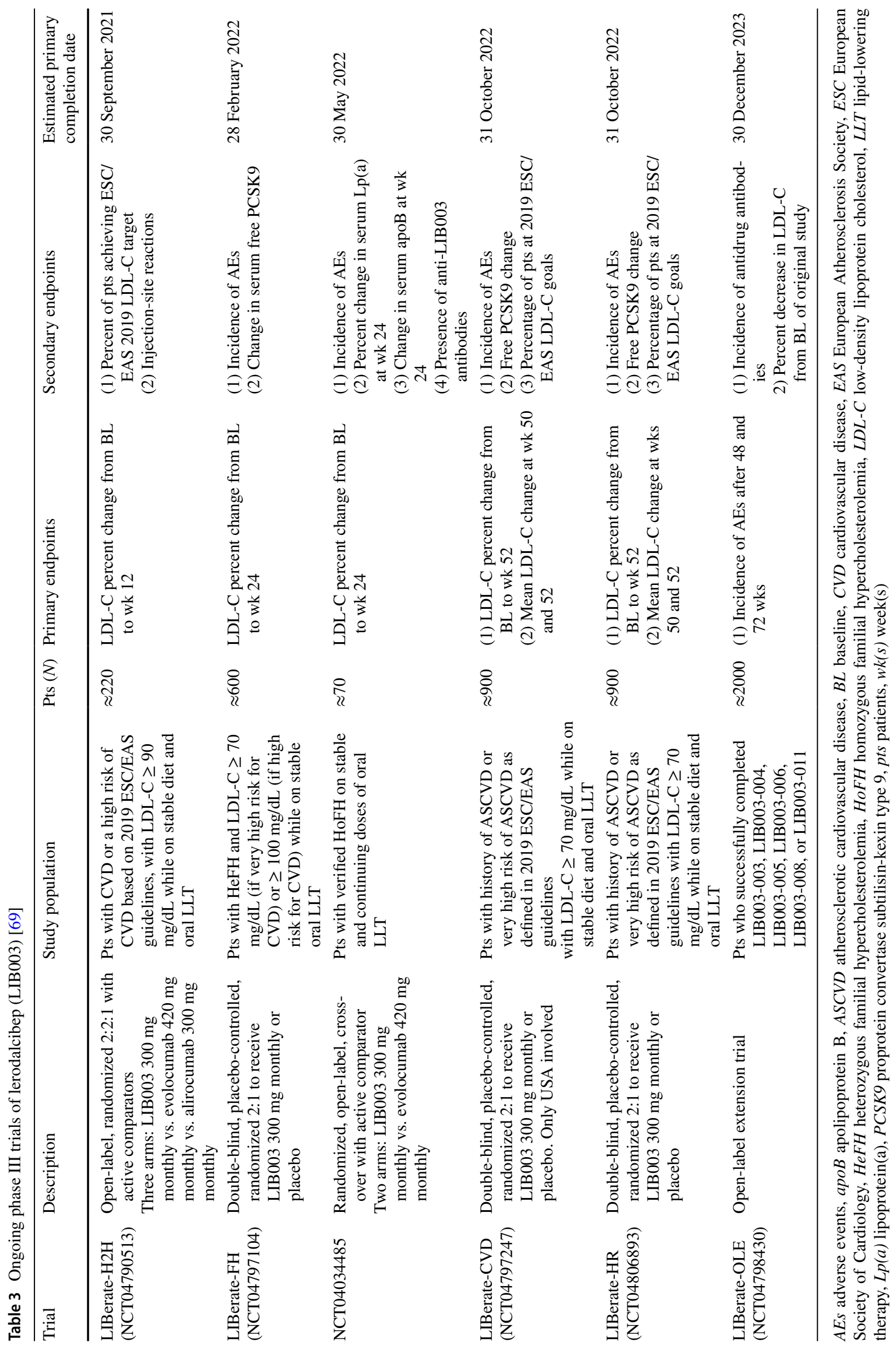




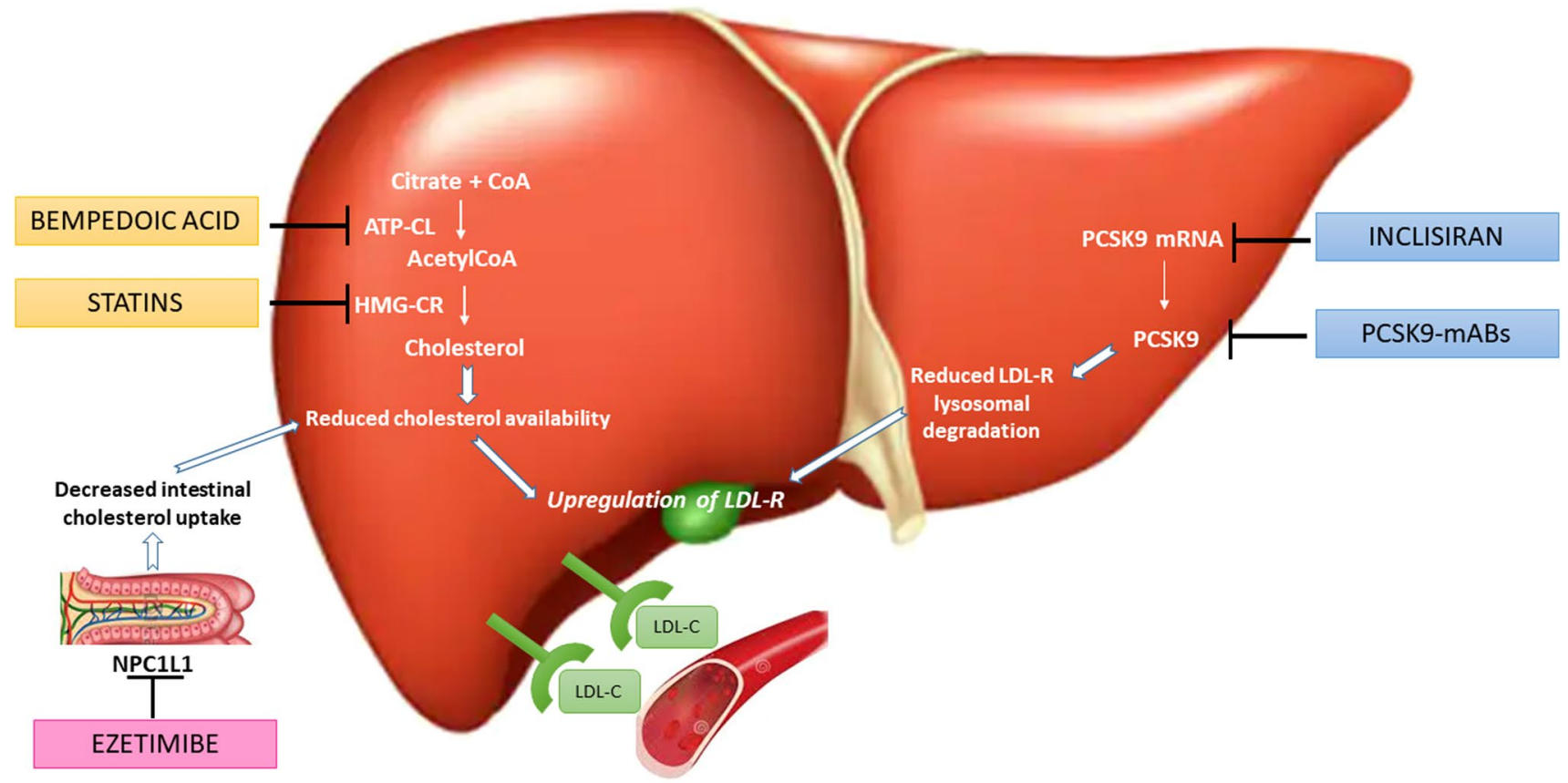

Fig. 1 Mechanisms of action of statins, ezetimibe, PCSK9i mAbs, bempedoic acid, and inclisiran. ATP-CL ATP citrate lyase, $C O A$ coenzyme A, $L D L-C$ low-density lipoprotein cholesterol, $L D L-R$ low-den-

sity lipoprotein receptor, $m A b s$ monoclonal antibodies, $m R N A$ messenger RNA, PCSK9 proprotein convertase subtilisin-kexin type 9

Table 4 Main characteristics of ezetimibe, PCSK9i monoclonal antibodies, bempedoic acid, and inclisiran

\begin{tabular}{|c|c|c|c|}
\hline Drug & Route, dose, and frequency of administration & Most common adverse reactions & $\begin{array}{l}\text { Mean } \\
\text { LDL-C } \\
\text { reduc- } \\
\text { tion }\end{array}$ \\
\hline Ezetimibe & Oral, $10 \mathrm{mg}$ daily & $\begin{array}{l}\text { Abdominal pain, flatulence, diarrhea, muscle-related } \\
\text { symptoms if given plus statins }\end{array}$ & $24 \%$ \\
\hline Evolocumab & $\begin{array}{l}\text { Subcutaneous injection, } 140 \mathrm{mg} \text { every } 2 \text { weeks or } 420 \\
\text { mg monthly }\end{array}$ & $\begin{array}{l}\text { Injection-site reactions, back pain, arthralgia, upper } \\
\text { respiratory symptoms }\end{array}$ & $59 \%$ \\
\hline Alirocumab & $\begin{array}{l}\text { Subcutaneous injection, } 75 / 150 \mathrm{mg} \text { every } 2 \text { weeks or } \\
300 \mathrm{mg} \text { monthly }\end{array}$ & $\begin{array}{l}\text { Injection-site reactions, upper respiratory symptoms, } \\
\text { itching }\end{array}$ & $55 \%$ \\
\hline Bempedoic acid & Oral, $180 \mathrm{mg}$ daily & Hyperuricemia and gout, anemia, arm and leg pain & $20 \%$ \\
\hline Inclisiran & $\begin{array}{l}\text { Subcutaneous injection, } 300 \mathrm{mg} \text { at time } 0 \text {, after } 3 \\
\text { months and then every } 6 \text { months }\end{array}$ & Injection-site reactions & $52 \%$ \\
\hline
\end{tabular}

LDL-C low-density lipoprotein cholesterol, PCSK9 proprotein convertase subtilisin-kexin type 9

cardiovascular risk and with good life expectancy. Among PCSK9i, based on RCTs, alirocumab appears to be the most studied after a recent ACS, whereas evolocumab is the only drug tested in patients with a lower eGFR of $20-30 \mathrm{~mL} / \mathrm{min} / 1.73 \mathrm{~m}^{2}$.

Some of the specific characteristics of patients included in the trials of each drug are represented in Fig. 2 and could be useful for a patient-tailored approach. In Fig. 3, we present a proposal of how to sequence these novel treatments during hospitalization for ACS. Indeed, hospitalization may also contribute to the implementation of LLT in clinical practice and may be an effective way of initiating treatment.

However, it is important to consider that the relative risk reduction in cardiovascular disease associated with LLT is associated with three variables: the absolute amount of LDL-C lowering, the baseline cardiovascular risk, and the duration of therapy. Specifically, the relative risk of major cardiovascular events and all-cause mortality per $38.7 \mathrm{mg} / \mathrm{dL}$ reduction in LDL-C has been calculated as 21 and $12 \%$ [6], respectively. Therefore, patients who start with higher levels 


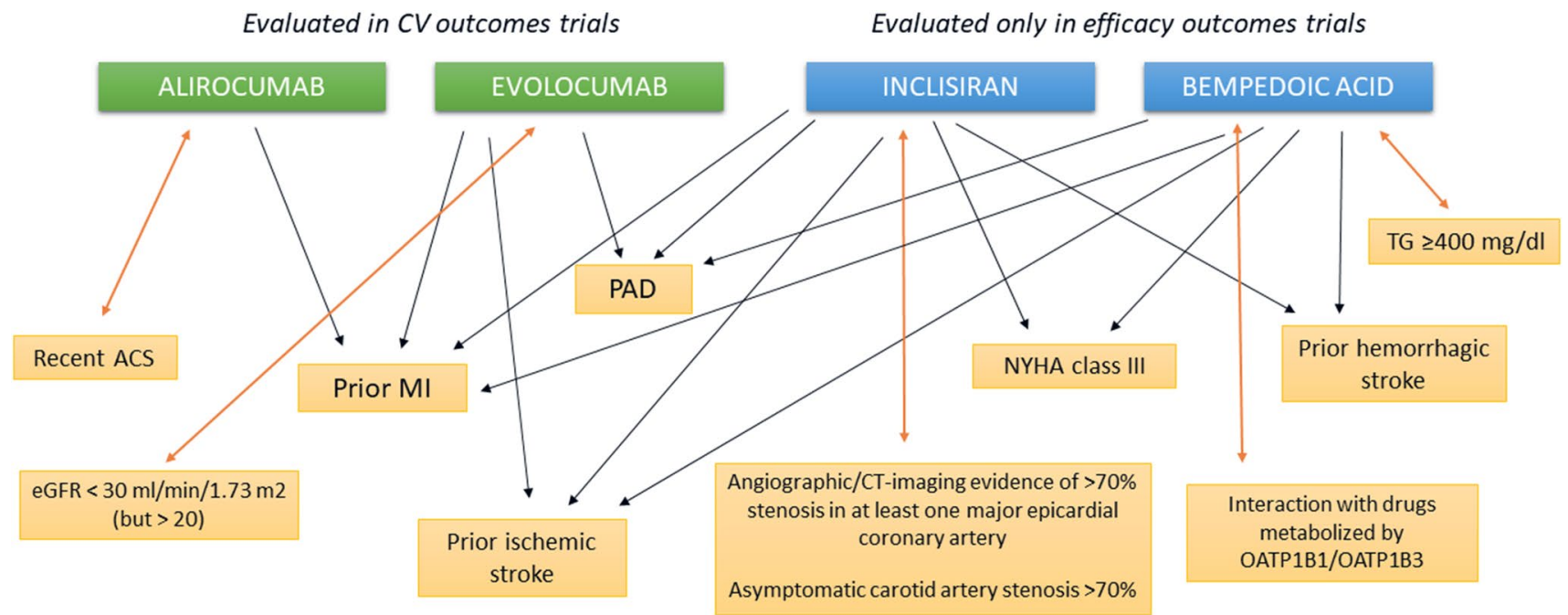

Fig. 2 The arrows connect every drug with specific characteristics of patients, on the basis of inclusion/exclusion criteria of corresponding RCTs. Orange arrows suggest exclusive links. ACS acute coronary syndrome, $C T$ computed tomography, $C V$ cardiovascular , $e G F R$ estimated glomerular filtration rate, $M I$ myocardial infarction, $N Y H A$ New York Heart Association, OATP organic anion-transporting peptide, $P A D$ peripheral artery disease, $T G$ triglyceride

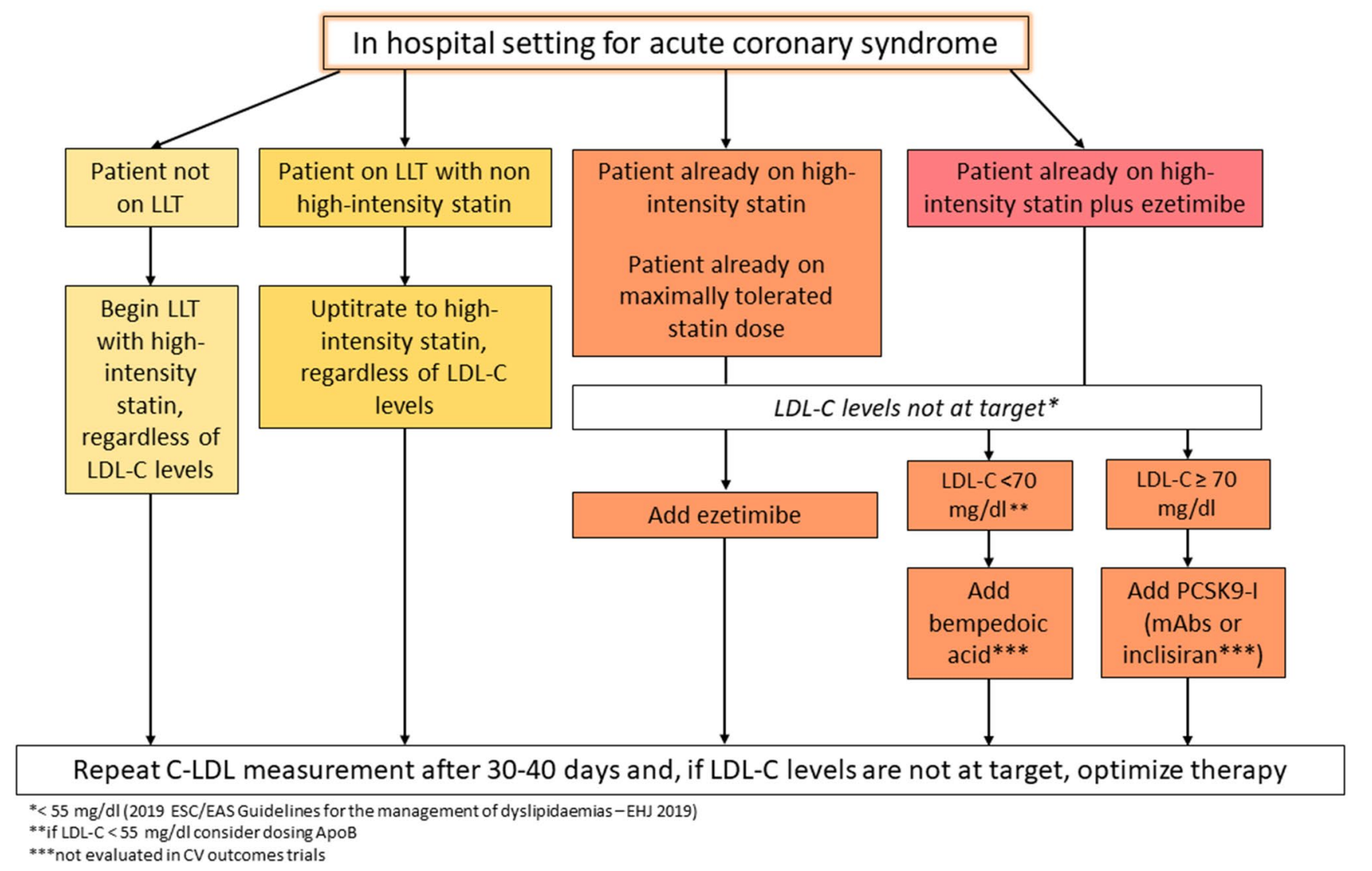

Fig. 3 Optimization of lipid-lowering therapy during hospitalization for acute coronary syndrome. $A p o B$ apolipoprotein B, EAS European Atherosclerosis Society, ESC European Society of Cardiology, $L D L$ -
$C$ low-density lipoprotein cholesterol, $L L T$ lipid-lowering therapy, $m A b s$ monoclonal antibodies, $P C S K 9 i$ proprotein convertase subtilisin-kexin type 9 inhibitor 
of LDL-C will experience greater cardiovascular risk reduction for any given percent decrease in LDL-C level. Furthermore, a recent analysis suggested that baseline LDL-C was a key driver of the magnitude of relative benefit derived from LLT with regard to reduction in all-cause and cardiovascular mortality, reporting the most meaningful results when LDL-C was $\geq 100 \mathrm{mg} / \mathrm{dL}$ [66]. In contrast, very high numbers needed to treat (NNTs) were observed for all-cause and cardiovascular mortality reduction with baseline LDL-C $<100 \mathrm{mg} / \mathrm{dL}$ [66]. A secondary analyses of the FOURIER trial showed that patients at high risk of cardiovascular disease (i.e., with recent ACS, residual multivessel coronary artery disease, more than one previous MI, PAD) may have a greater relative risk reduction with LLT [67]. Importantly, the combination of higher baseline risk and greater relative risk reduction led to a greater absolute risk reduction at 3 years [68].

Another important factor is the duration of treatment, given that significant heterogeneity has been described in the benefit from LLT depending on the length of treatment. In the first year of therapy, the relative risk reduction of cardiovascular events per $38.7 \mathrm{mg} / \mathrm{dL}$ reduction in LDL-C plasma levels was only $9 \%$, subsequently growing to $22 \%$, $24 \%$, and so on for each additional year of therapy [68].

Therefore, patients with higher cardiovascular risk, LDL-C baseline levels $\geq 100 \mathrm{mg} / \mathrm{dL}$, and greater life expectancy represents the subgroup most likely to experience the largest absolute risk reductions, with smaller NNTs to prevent a cardiovascular event. Given that PCSK9i (mAbs or inclisiran) are currently expensive, treatment should be reserved for selected populations to meet conventional costeffectiveness standards; alternatively, for lower risk, significant cost reductions would be needed.

\section{Conclusions}

Given the global high burden of ASCVD, LLT is one of the most promising fields of cardiovascular research. Besides statins, ezetimibe, and PCSK9 mAbs, whose effects on cardiovascular outcomes are well-known, recently approved inclisiran and bempedoic acid, with their different efficacy and administration routes and times, will further expand the available pharmacologic armamentarium, enabling the clinician to decrease residual risk related to LDL-C.

\section{Declarations}

Funding Open access funding provided by Università degli Studi di Trieste within the CRUI-CARE Agreement.

Conflict of interest Maddalena Rossi, Enrico Fabris, Davide Barbisan, Laura Massa, and Gianfranco Sinagra have no conflicts of interest that are directly relevant to the content of this article.
Availability of data and material Not applicable.

Ethics approval Not applicable.

Consent Not applicable.

Author contributions All the listed authors contributed to the work. In particular, Dr. MR, Dr. EF, and Dr. DB drafted the manuscript; Dr. Massa and Professor GS supervised the project and reviewed the work.

Open Access This article is licensed under a Creative Commons Attribution-NonCommercial 4.0 International License, which permits any non-commercial use, sharing, adaptation, distribution and reproduction in any medium or format, as long as you give appropriate credit to the original author(s) and the source, provide a link to the Creative Commons licence, and indicate if changes were made. The images or other third party material in this article are included in the article's Creative Commons licence, unless indicated otherwise in a credit line to the material. If material is not included in the article's Creative Commons licence and your intended use is not permitted by statutory regulation or exceeds the permitted use, you will need to obtain permission directly from the copyright holder. To view a copy of this licence, visit http://creativecommons.org/licenses/by-nc/4.0/.

\section{References}

1. Mathers CD, Loncar D. Projections of Global Mortality and Burden of Disease from 2002 to 2030. PLoS Med. 2006;3(11): e442. https://doi.org/10.1371/journal.pmed.0030442.

2. Centre for Economics and Business Research. The economic cost of cardiovascular disease from 2014-2020 in six European economies. 2020.

3. Lowering blood cholesterol to prevent heart disease. NIH Consensus Development Conference statement. Arteriosclerosis 5(4):404-12. http://www.ncbi.nlm.nih.gov/pubmed/3839391.

4. Yusuf S, et al. Effect of potentially modifiable risk factors associated with myocardial infarction in 52 countries (the INTERHEART study): case-control study. Lancet (Lond, Engl). 2004;364(9438):37-52. https://doi.org/10.1016/S0140-6736(04) 17018-9.

5. Sinning D, Landmesser U. Low-density lipoprotein-cholesterol lowering strategies for prevention of atherosclerotic cardiovascular disease: focus on siRNA treatment targeting PCSK9 (Inclisiran). Curr Cardiol Rep. 2020;22(12):176. https://doi.org/10. 1007/s11886-020-01427-6.

6. Baigent C, Keech A, Kearney PM, et al. Efficacy and safety of cholesterol-lowering treatment: prospective meta-analysis of data from 90056 participants in 14 randomised trials of statins. Lancet. 2005;366(9493):1267-78. https://doi.org/10.1016/S01406736(05)67394-1.

7. Mach F, et al. 2019 ESC/EAS Guidelines for the management of dyslipidaemias: lipid modification to reduce cardiovascular risk. Eur Heart J. 2020;41(1):111-88. https://doi.org/10.1093/eurheartj/ ehz455.

8. Ference BA, et al. Low-density lipoproteins cause atherosclerotic cardiovascular disease. 1. Evidence from genetic, epidemiologic, and clinical studies. A consensus statement from the European Atherosclerosis Society Consensus Panel. Eur Heart J. 2017;38(32):2459-72. https://doi.org/10.1093/eurheartj/ehx144.

9. Cholesterol Treatment Trialists' (CTT) Collaboration. Efficacy and safety of more intensive lowering of LDL cholesterol: a metaanalysis of data from 170000 participants in 26 randomised trials. 
Lancet. 2010;376(9753):1670-1681. doi: https://doi.org/10.1016/ S0140-6736(10)61350-5.

10. Brown M, Goldstein J. A receptor-mediated pathway for cholesterol homeostasis. Science (80-). 1986;232(4746):34-47. https:// doi.org/10.1126/science. 3513311 .

11. Collins R, Reith C, Emberson J, Armitage J, Baigent C. Interpretation of the evidence for the efficacy and safety of statin therapy. Lancet (Lond, Engl). 2016. https://doi.org/10.1016/S01406736(18)31942-1.

12. Zhang $\mathrm{H}$, et al. Discontinuation of statins in routine care settings. Ann Intern Med. 2013;158(7):526. https://doi.org/10.7326/00034819-158-7-201304020-00004.

13. Jones PH, Nair R, Thakker KM. Prevalence of dyslipidemia and lipid goal attainment in statin-treated subjects from 3 data sources: a retrospective analysis. J Am Heart Assoc. 2012. https://doi.org/ 10.1161/JAHA.112.001800.

14. Davis HR, et al. Niemann-Pick C1 Like 1 (NPC1L1) is the intestinal phytosterol and cholesterol transporter and a key modulator of whole-body cholesterol homeostasis. J Biol Chem. 2004;279(32):33586-92. https://doi.org/10.1074/jbc.M4058 17200.

15. Pearson TA, et al. Pooled analyses of effects on C-reactive protein and low density lipoprotein cholesterol in placebo-controlled trials of ezetimibe monotherapy or ezetimibe added to baseline statin therapy. Am J Cardiol. 2009;103(3):369-74. https://doi.org/10. 1016/j.amjcard.2008.09.090.

16. Rosseb $\varnothing \mathrm{AB}$, et al. Intensive lipid lowering with simvastatin and ezetimibe in aortic stenosis. N Engl J Med. 2008;359(13):134356. https://doi.org/10.1056/NEJMoa0804602.

17. Baigent $\mathrm{C}$, et al. The effects of lowering LDL cholesterol with simvastatin plus ezetimibe in patients with chronic kidney disease (Study of Heart and Renal Protection): a randomised placebocontrolled trial. Lancet. 2011;377(9784):2181-92. https://doi.org/ 10.1016/S0140-6736(11)60739-3.

18. Cannon CP, et al. Ezetimibe added to statin therapy after acute coronary syndromes. N Engl J Med. 2015;372(25):2387-97. https://doi.org/10.1056/NEJMoa1410489.

19. Cannon CP, Khan I, Klimchak AC, Reynolds MR, Sanchez RJ, Sasiela WJ. Simulation of lipid-lowering therapy intensification in a population with atherosclerotic cardiovascular disease. JAMA Cardiol. 2017;2(9):959. https://doi.org/10.1001/jamacardio.2017. 2289.

20. Steen DL, Khan I, Ansell D, Sanchez RJ, Ray KK. Retrospective examination of lipid-lowering treatment patterns in a real-world high-risk cohort in the UK in 2014: comparison with the National Institute for Health and Care Excellence (NICE) 2014 lipid modification guidelines. BMJ Open. 2017;7(2): e013255. https://doi. org/10.1136/bmjopen-2016-013255.

21. Reyes-Soffer G, et al. Effects of PCSK9 inhibition with alirocumab on lipoprotein metabolism in healthy humans. Circulation. 2017;135(4):352-62. https://doi.org/10.1161/CIRCULATIO NAHA.116.025253.

22. Watts GF, et al. Factorial effects of evolocumab and atorvastatin on lipoprotein metabolism. Circulation. 2017;135(4):338-51. https://doi.org/10.1161/CIRCULATIONAHA.116.025080.

23. Ridker PM, et al. Lipid-reduction variability and antidrug-antibody formation with bococizumab. N Engl J Med. 2017;376(16):1517-26. https://doi.org/10.1056/NEJMoa1614062.

24. Robinson JG, et al. Efficacy and safety of alirocumab in reducing lipids and cardiovascular events. N Engl J Med. 2015;372(16):1489-99. https://doi.org/10.1056/NEJMoa1501031.

25. Sabatine MS, et al. Evolocumab and clinical outcomes in patients with cardiovascular disease. N Engl J Med. 2017;376(18):171322. https://doi.org/10.1056/NEJMoa1615664.
26. Schwartz GG, et al. Alirocumab and cardiovascular outcomes after acute coronary syndrome. N Engl J Med. 2018;379(22):2097-107. https://doi.org/10.1056/NEJMoa1801174.

27. Ference BA, Cannon CP, Landmesser U, Lüscher TF, Catapano AL, Ray KK. Reduction of low density lipoprotein-cholesterol and cardiovascular events with proprotein convertase subtilisin-kexin type 9 (PCSK9) inhibitors and statins: an analysis of FOURIER, SPIRE, and the Cholesterol Treatment Trialists Collaboration. Eur Heart J. 2018;39(27):2540-5. https://doi.org/10.1093/eurheartj/ ehx450.

28. Giugliano RP, et al. Clinical efficacy and safety of evolocumab in high-risk patients receiving a statin. JAMA Cardiol. 2017;2(12):1385. https://doi.org/10.1001/jamacardio.2017.3944.

29. Giugliano RP, et al. Clinical efficacy and safety of achieving very low LDL-cholesterol concentrations with the PCSK9 inhibitor evolocumab: a prespecified secondary analysis of the FOURIER trial. Lancet. 2017;390(10106):1962-71. https://doi.org/10.1016/ S0140-6736(17)32290-0.

30. Giugliano RP, et al. Cognitive function in a randomized trial of evolocumab. N Engl J Med. 2017;377(7):633-43. https://doi.org/ 10.1056/NEJMoa1701131.

31. Stoekenbroek RM, Kallend D, Wijngaard PL, Kastelein JJ. Inclisiran for the treatment of cardiovascular disease: the ORION clinical development program. Fut Cardiol. 2018;14(6):433-42. https://doi.org/10.2217/fca-2018-0067.

32. Azari S, et al. Cost-effectiveness analysis of PCSK9 inhibitors in cardiovascular diseases: a systematic review. Heart Fail Rev. 2020;25(6):1077-88. https://doi.org/10.1007/ s10741-019-09874-2.

33. Colivicchi F, et al. Updated clinical evidence and place in therapy of bempedoic acid for hypercholesterolemia. J Cardiovasc Med. 2020. https://doi.org/10.2459/JCM.0000000000001108.

34. Pinkosky SL, et al. Liver-specific ATP-citrate lyase inhibition by bempedoic acid decreases LDL-C and attenuates atherosclerosis. Nat Commun. 2016;7(1):13457. https://doi.org/10.1038/ncomm s13457.

35. Ballantyne CM, et al. Efficacy and safety of bempedoic acid added to ezetimibe in statin-intolerant patients with hypercholesterolemia: a randomized, placebo-controlled study. Atherosclerosis. 2018;277:195-203. https://doi.org/10.1016/j.atherosclerosis.2018. 06.002 .

36. Laufs $\mathrm{U}$, et al. Efficacy and safety of bempedoic acid in patients with hypercholesterolemia and statin intolerance. J Am Heart Assoc. 2019. https://doi.org/10.1161/JAHA.118.011662.

37. Ray KK, et al. Safety and efficacy of bempedoic acid to reduce LDL cholesterol. N Engl J Med. 2019;380(11):1022-32. https:// doi.org/10.1056/NEJMoa1803917.

38. Goldberg AC, et al. Effect of bempedoic acid vs placebo added to maximally tolerated statins on low-density lipoprotein cholesterol in patients at high risk for cardiovascular disease. JAMA. 2019;322(18):1780. https://doi.org/10.1001/jama.2019.16585.

39. Khan MU, Khan MZ, Munir MB, Balla S, Khan SU. Meta-analysis of the safety and efficacy of bempedoic acid. Am J Cardiol. 2020;131:130-2. https://doi.org/10.1016/j.amjcard.2020.06.028.

40. European Medicine Agency. https://www.ema.europa.eu/en/medic ines/human/EPAR/nilemdo.

41. European Medicine Agency. https://www.ema.europa.eu/en/medic ines/human/EPAR/nustendi.

42. Preiss D, Tobert JA, Hovingh GK, Reith C. Lipid-modifying agents, from statins to PCSK9 inhibitors. J Am Coll Cardiol. 2020;75(16):1945-55. https://doi.org/10.1016/j.jacc.2019.11.072.

43. Khvorova A. Oligonucleotide therapeutics-a new class of cholesterol-lowering drugs. N Engl J Med. 2017;376(1):4-7. https:// doi.org/10.1056/NEJMp1614154. 
44. Raal FJ, et al. Inclisiran for the treatment of heterozygous familial hypercholesterolemia. N Engl J Med. 2020;382(16):1520-30. https://doi.org/10.1056/NEJMoa1913805.

45. Ray KK, et al. Two phase 3 trials of inclisiran in patients with elevated LDL cholesterol. N Engl J Med. 2020;382(16):1507-19. https://doi.org/10.1056/NEJMoa1912387.

46. https://www.thepharmaletter.com/article/icer-takes-hard-line-onnew-cholesterol-drugs.

47. European Medicine Agency. https://www.ema.europa.eu/en/medic ines/human/EPAR/leqvio.

48. Cuchel M, et al. Homozygous familial hypercholesterolaemia: new insights and guidance for clinicians to improve detection and clinical management. A position paper from the Consensus Panel on Familial Hypercholesterolaemia of the European Atherosclerosis Society. Eur Heart J. 2014;35(32):2146-57. https://doi.org/10. 1093/eurheartj/ehu274.

49. Jia X, Liu J, Mehta A, Ballantyne CM, Virani SS. Lipid-lowering biotechnological drugs: from monoclonal antibodies to antisense therapies-a clinical perspective. Cardiovasc Drugs Ther. 2020. https://doi.org/10.1007/s10557-020-07082-x.

50. Cuchel M, et al. Efficacy and safety of a microsomal triglyceride transfer protein inhibitor in patients with homozygous familial hypercholesterolaemia: a single-arm, open-label, phase 3 study. Lancet. 2013;381(9860):40-6. https://doi.org/10.1016/S01406736(12)61731-0.

51. https://www.ema.europa.eu/documents/product-information/lojux ta-epar-product-information_en.pdf.

52. Tomlinson B, Chan P, Zhang Y, Lam CWK. Efficacy and safety of add on therapies in patients with hypercholesterolemia undergoing statin therapy. Expert Opin Pharmacother. 2020;21(17):2137-51. https://doi.org/10.1080/14656566.2020.1801638.

53. Barter PJ, et al. Effects of torcetrapib in patients at high risk for coronary events. N Engl J Med. 2007;357(21):2109-22. https:// doi.org/10.1056/NEJMoa0706628.

54. Tall AR, Rader DJ. Trials and tribulations of CETP inhibitors. Circ Res. 2018;122(1):106-12. https://doi.org/10.1161/CIRCR ESAHA.117.311978.

55. Effects of anacetrapib in patients with atherosclerotic vascular disease. N Engl J Med. 2017;377(13):1217-1227. doi: https://doi. org/10.1056/NEJMoa1706444.

56. Merck. Merck provides update on anacetrapib development program. Published on October 11, 2017.
57. Hovingh GK, et al. Cholesterol ester transfer protein inhibition by TA-8995 in patients with mild dyslipidaemia (TULIP): a randomised, double-blind, placebo-controlled phase 2 trial. Lancet. 2015;386(9992):452-60. https://doi.org/10.1016/S0140-6736(15) 60158-1.

58. https://clinicaltrials.gov/ct2/show/NCT04753606.

59. https://clinicaltrials.gov/ct2/show/NCT04770389.

60. Ding Q, et al. Permanent alteration of PCSK9 with in vivo CRISPR-Cas9 genome editing. Circ Res. 2014;115(5):488-92. https://doi.org/10.1161/CIRCRESAHA.115.304351.

61. Landlinger $\mathrm{C}$, et al. The AT04A vaccine against proprotein convertase subtilisin/kexin type 9 reduces total cholesterol, vascular inflammation, and atherosclerosis in APOE*3Leiden.CETP mice. Eur Heart J. 2017;38(32):2499-507. https://doi.org/10.1093/eurhe $\operatorname{artj} / \mathrm{eh} \times 260$.

62. https://pace-cme.org/2019/05/28/novel-pcsk9-inhibitor-yieldssafe-and-effective-ldl-c-lowering/.

63. RZ Evan, A Stein, T Turner, DJ Kereiakes, B Butcher, P Mangu. Abstract 17222: safety, tolerability and LDL-C reduction with LIB003 a novel anti-PCSK9 recombinant fusion protein: results of open-label extension phase 2B study. Circ. 2019;140A17222.

64. Momtazi-Borojeni AA, Jaafari MR, Badiee A, Sahebkar A. Longterm generation of antiPCSK9 antibody using a nanoliposomebased vaccine delivery system. Atherosclerosis. 2019;283:69-78. https://doi.org/10.1016/j.atherosclerosis.2019.02.001.

65. Sahebkar A, Momtazi-Borojeni AA, Banach M. PCSK9 vaccine: so near, yet so far! Eur Heart J. 2021. https://doi.org/10.1093/ eurheartj/ehab299.

66. Navarese EP, et al. Association between baseline LDL-C level and total and cardiovascular mortality after LDL-C lowering. JAMA. 2018;319(15):1566. https://doi.org/10.1001/jama.2018.2525.

67. Sabatine MS, et al. Clinical benefit of evolocumab by severity and extent of coronary artery disease. Circulation. 2018;138(8):75666. https://doi.org/10.1161/CIRCULATIONAHA.118.034309.

68. Sabatine MS. PCSK9 inhibitors: clinical evidence and implementation. Nat Rev Cardiol. 2019;16(3):155-65. https://doi.org/10. 1038/s41569-018-0107-8.

69. Evidence-Based Medicine DataLab, University of Oxford. FDAA TrialsTracker. https://fdaaa.trialstracker.net/sponsor/lib-therapeuti cs-1lc/. 